\title{
Evaluation of Optic Nerve Head Parameters and Retinal Nerve Fiber Layer Thickness in Axial Myopia Using SD OCT
}

\author{
Heba Radi AttaAllah • Ismail Ahmed Nagib Omar • Ahmed Shawkat Abdelhalim
}

Received: April 3, 2017 / Published online: June 5, 2017

(C) The Author(s) 2017. This article is an open access publication

\begin{abstract}
Introduction: The purpose of this research was to study the effect of increased axial myopia, in non-glaucomatous eyes, and its correlation with ONH parameters, and RNFL thickness, using Cirrus HD 4000 SD-OCT.

Methods: The myopia group included 86 eyes of 86 patients, while the control group involved 92 eyes of 92 patients, attending the Ophthalmology Outpatient Clinic in Minia University Hospital, between November 2013 and March 2015. ONH parameters and peripapillary RNFL thickness measurement were evaluated by using SD-OCT, selecting the standard optic disc cube $200 \times 200$.

regarding disc area $(p=0.01)$, rim area $(p=0.001)$, vertical C/D $(p=0.01)$, average C/D ratio $(p=0.001)$, average and temporal RNFL thickness ( $p=0.0001, p=0.001$, respectively).

Conclusion: A significant difference was found between highly myopic non-glaucomatous eyes and the control group, regarding $\mathrm{ONH}$ parameters and RNFL thickness as measured by SD-OCT. As OCT magnification adjusted ONH parameters were larger, global and the temporal RNEL were thicker in the myopia group, those magnification adjusted parameters helped in an accurate evaluation of ONH and RNFL in highly myopic eyes, in order to avoid misdiagnosis of glaucoma in such eyes.
\end{abstract} Results: The mean age of the myopia group was $(36.55 \pm 9.44)$, the mean spherical equivalent was $(-12.70 \pm 3.87 \mathrm{D})$, and the mean axial length was $(27.88 \pm 1.92 \mathrm{~mm})$. The control group had mean age of $(34.82 \pm 8.87)$, mean spherical equivalent of $-0.65 \pm 0.41 \mathrm{D}$, and mean AL of $(22.16 \pm 0.82)$. A significant difference was reported between the two groups,

Enhanced content To view enhanced content for this article go to http://www.medengine.com/Redeem/ OF48F0607755EF5C.

H. R. AttaAllah $(\bowtie) \cdot$ I. A. N. Omar .

A. S. Abdelhalim

Ophthalmology Department, Minia University

Hospital, Faculty of Medicine, Minia University,

El-Minya, Egypt

e-mail: radi_heba@yahoo.com
Keywords: Myopia; ONH parameters; RNFL thickness

\section{INTRODUCTION}

Optic nerve head (ONH) and retinal nerve fiber layer (RNFL), show a remarkable variation within the normal population, and are not uniform in appearance, as they are biological structures [1]. The introduction of recent imaging modalities such as optical coherence tomography (OCT), Heidelberg Retinal Tomography (HRT), and confocal scanning laser ophthalmoscopy (CSLO), added valuable information for clinical assessment of the $\mathrm{ONH}$, and RNFL [2,3]. The determination of $\mathrm{ONH}$ 
quantitative parameters, such as the area of the optic disc and cup and their ratio, helped in the diagnosis and monitoring of diseases affecting the optic nerve [4].

The impact of refractive errors on $\mathrm{ONH}$ parameters is controversial. Some studies found that the refractive errors had only a minimal effect on measurements of optic nerve parameters among the different modalities [5]. While, others have reported stronger associations between optic nerve parameters and high refractive errors [6]. ONH was abnormally large in highly myopic $(>-8.0 \mathrm{D})$ eyes, and abnormally small in highly hyperopic eyes $(>+4 \mathrm{D})$ [7].

Thinning of RNFL is a very good indicator of glaucoma. Misdiagnosis of glaucoma can occur on measuring RNFL thickness without assessment of the refractive state, especially in myopic eyes [8].

The aim of this study is to evaluate the effect of increased axial myopia, in non-glaucomatous eyes, and its correlation with the $\mathrm{ONH}$ parameter, and RNFL thickness using SD-OCT.

\section{METHODS}

This study is a cross-sectional study, it included 86 eyes of 86 highly myopic individuals, and 92 eyes of 92 normal control persons; all participants were Egyptian, who were attending the Ophthalmology out patient clinic, at Minia University hospital, between November 2013 and March 2015. The study gained approval from the Local Research Ethics Committee, Faculty of Medicine, Minia University Hospitals. Informed consent was obtained from all patients for being included in this study.

All patients included in the myopia group, had their spherical equivalent (SE) $\geq-6.00 \mathrm{D}$, and axial length (AL) $\geq 26 \mathrm{~mm}$. While all patients included in the control group had their spherical equivalent (SE) ranging between -1.00 and $+1.00 \mathrm{D}$, and axial length ranging between 21.50 and $23.50 \mathrm{~mm}$. All eyes with: glaucoma, previous intraocular surgery, optic nerve affection (papilledema, optic atrophy, ischemic optic neuropathy, compressive optic neuropathy, demyelinating optic neuropathy (multiple sclerosis) or congenital abnormalities of the disc such as: tilted disc, hypoplastic disc, etc.), peripapillary atrophy (extending to $\geq 3.4 \mathrm{~mm}$ around the disc, that affects the peripapillary RNFL measurements), retinal vascular disorders or macular disorders, and media opacities that may affect scans' signal strength, were excluded from both groups.

Glaucoma was excluded by: absence of glaucomatous disc changes, normal visual field testing by 30-2 SITA (Swedish interactive threshold algorithm) standard automated visual field test, and IOP less than $20 \mathrm{mmHg}$.

All studied individuals were subjected to the following ophthalmic evaluation: visual acuity assessment, anterior segment evaluation by slit-lamp biomicroscopy, fundus examination and optic nerve head $(\mathrm{ONH})$ evaluation by the use of a 90-D Volk lens, intraocular pressure (IOP) measurement by Tono-Pen ${ }^{\circledR}$ XL Applanation Tonometer (USA), cycloplegic refraction using Nidek autoref/keratometer (LS 900, HAAGSTREIT DIAGNOSTICS, Switzerland), refractive error was expressed as the spherical equivalent (SE), axial length (AL) measurement was using al Quantel medical Aviso (A\&B scan Ophthalmic Echography, 2008, France), visual field analysis: the 30-2 SITA standard test was performed using (Humphrey Visual Field Analyzer; Model HFA-745i, Carl Zeiss Meditec).

OCT examination after pupillary dilatation, using Cirrus HD OCT model 4000 (Cirrus HD-OCT, software version 4.0.1.3; Carl Zeiss Meditec Inc, Dublin, USA), ONH analysis and peripapillary RNFL thickness measurments in four quadrants were obtained using the standard optic disc cube $200 \times 200$ acquisition protocol. Good quality scans were selected and used for analysis, which include: scans with signal strength $\geq 6$, without blinking artifacts or RNFL discontinuity, and absence of RNFL algorithm segmentation failure. Only one randomly selected eye was examined for each patient. All OCT examinations were performed by one observer (H.R.). Data about optic nerve head parameters and retinal nerve fiber layer thickness were collected and analyzed.

Magnification-adjusted OCT parameters were calculated in the myopia group; as ocular magnification can affect OCT measurements in such highly myopic eyes. The following formula 
$t=p \times q \times s$, is used to express the relationship between the actual fundus dimension measurements and the measurements obtained by an imaging system, where $t$ is the actual fundus dimension, $p$ is the magnification factor for the imaging system, $q$ is the magnification factor related to the eye and $s$ is the measurement obtained by an imaging system [9]. As the OCT system has a $p$ value of 3.382 [10], the formula for obtaining the $q$ value (magnification factor) for the eye is $q=0.01306 \times(\mathrm{AL}-1.82)$ [11].

Therefore, the adjusted parameters for area (disc area and rim area), were calculated using the following formula:

$$
\begin{aligned}
\text { Adjusted area }= & 3.382^{2} \times 0.01306^{2} \times(\mathrm{AL} \\
& -1.82)^{2} \times \text { measured area } .
\end{aligned}
$$

The magnification adjusted average RNFL thickness, was calculated using the following formula: $t=p \times q \times s$.

\section{Adjusted average RNFL thickness} $=3.382 \times 0.01306 \times(\mathrm{AL}-1.82)$ $\times$ measured average RNFL thickness,

where $t=$ adjusted average RNFL thickness, $p=3.382, \quad q=0.01306 \times(\mathrm{AL}-1.82), \quad$ and $s=$ measured average RNFL thickness.

\section{Statistical Analysis}

Data entry and analysis were all done with Statistical Package for Social Sciences (SPSS) version 19. Quantitative data were presented by mean and standard deviation, while qualitative data were presented as frequency distribution. Student $t$ test was used to compare two means. Karl Pearson's correlation coefficient (r) was also used. The probability of less than 0.05 was used as a cut off point for all significant tests.

\section{RESULTS}

The myopia group included 86 eyes of 86 patients (37 males and 49 females), with mean age of $36.55 \pm 9.44$ years old (22-41), the mean spherical equivalent (MSE) was $-12.70 \pm 3.87 \mathrm{D}$
( -7.00 to -20.00$)$ and the mean axial length (AL) was $27.88 \pm 1.92 \mathrm{~mm}$ (26.14-29.50).

Regarding the control group, it involved 92 eyes of 92 patients (36 males and 56 females), with mean age of $34.82 \pm 8.87$ years $(20-45)$, they had MSE of $-0.65 \pm 0.41(-0.25$ to +1.00$)$, and mean $\mathrm{AL}$ of $22.16 \pm 0.82 \mathrm{~mm}$ (21.95-23.40).

The mean IOP in the myopia was $14.90 \pm 1.73 \mathrm{mmHg} \quad(12-18), \quad$ and $14.78 \pm 1.1 \mathrm{mmHg}(11-17)$ in the control group. We found no statistically significant difference between both groups regarding the age or IOP.

Analysis of the RNFL thickness revealed that the thickest quadrant was the superior in the myopia group, but it was the inferior in the control group, while the thinnest was the nasal and the temporal, respectively (Table 2 ).

\section{DISCUSSION}

Certain anatomical changes that occur in longer axial length eyes, such as globe elongation, scleral widening, and subsequently enlargement of the lamina cribrosa, result in larger disc areas in such eyes. The use of magnification-adjustment can correct disc and rim areas measurements in those eyes.

Some studies using the Littman equation for magnification adjustment indicate that a magnification adjustment formula based on axial length is more accurate than other factors [12], and applied the formulas to global RNFL thickness, and disc area measurements in myopic subjects [13].

In the current study, regarding the optic disc and neuroretinal rim areas, a statistically significant increase was found in the myopia group as compared with the control group (Table 1), as the highly myopic eyes obtained a significantly larger disc, and rim areas. These results were consistent with previous study, which showed significant correlations between neuroretinal rim and disc area, and myopic refractive error, depending on optic disc colour photographs [14].

In the current study, the mean magnification-adjusted disc area, in the myopia group, 
Table 1 Comparison between $\mathrm{ONH}$ parameters in both groups

\begin{tabular}{lccc}
\hline & Myopia group & Control group & $\boldsymbol{p}$ value \\
\hline Disc area & $3.29 \pm 0.66(1.56-5.38)$ & $1.97 \pm 0.23(1.58-2.37)$ & 0.01 \\
Rim area & $2.51 \pm 0.48(1.42-3.9)$ & $1.47 \pm 0.19(1.03-1.87)$ & 0.001 \\
Average C/D & $0.56 \pm 0.119(0.27-0.65)$ & $0.37 \pm 0.082(0.31-0.48)$ & 0.001 \\
Vertical C/D & $0.55 \pm 0.117(0.25-0.66)$ & $0.39 \pm 0.086(0.30-0.47)$ & 0.01 \\
\hline
\end{tabular}

$O N H$ optic nerve head, $C / D$ cup/disc ratio

was $3.29 \pm 0.66 \mathrm{~mm}^{2}$, which was comparable with the Hsu et al. study, as their mean optic disc area, using magnification-adjusted OCT measurements, was $3.30 \pm 0.70 \mathrm{~mm}$ [15]. Also, this applies for the mean magnification-adjusted rim area, in the highly myopic group, which was $2.51 \pm 0.48 \mathrm{~mm}$, and was consistent with the other study result, as it was $2.45 \pm 0.69 \mathrm{~mm}$.

Another study, showed a smaller mean disc area, and rim area than current study results, as it was $2.07 \pm 0.45$, and $1.3 \pm 0.22 \mathrm{~mm}^{2}$, respectively, with magnification effect correction, their study included Korean participants with lower degrees of myopia, MSE $-3.28 \pm 2.27 \mathrm{D}$ (range -9.38 to -0.13 ), and shorter $\mathrm{AL}$ $25.03 \pm 1.27 \mathrm{~mm}$ (range 22.84-28.60), factors that may contribute to the discrepancy between the two studies' results [16].

The average $\mathrm{C} / \mathrm{D}$ ratio value was $0.56 \pm 0.119 \mathrm{~mm}$ in the myopia group, which was significantly larger than that of the control group $(0.37 \pm 0.08 \mathrm{~mm})$ (Table 1$)$. Also, the vertical C/D was significantly of higher values in the myopia group $(0.55 \pm 0.117 \mathrm{~mm})$, while in the control group it was $0.39 \pm 0.08 \mathrm{~mm}$ (Table 1).
In the current study, average magnification-adjusted RNFL was thicker in the myopic group than in the control group, as it was $(109.21 \pm 7.38 \mu \mathrm{m}), \quad(96.30 \pm 4.91 \mu \mathrm{m}) \quad$ respectively, $(p=0.0001)$ (Table 2$)$. This was consistent with previous reports that magnification-adjusted OCT measurements of global RNFL thickness was thicker than normal, in eyes with axial myopia $[13,15]$.

Temporal RNFL was significantly thicker than normal, in the myopia group. Which can be explained by the redistribution of nerve fiber layer in highly myopic eyes, that causes temporal retinal dragging and increased temporal RNFL thickness, also it increases the posterior staphyloma height in the nasal fovea area $[17,18]$.

No significant difference between myopic and control eyes, regarding OCT measurements of the vertical RNFLs (superior and inferior RNFL), which is useful for differential diagnosis between myopia and glaucoma. Kang and colleague [13], reported that superior and inferior temporal RNFL thicknesses (measured at the 11 and 7 o'clock positions, respectively) do not differ significantly between the highly myopic

Table 2 Comparison between RNFL thicknesses in both groups

\begin{tabular}{lccc}
\hline & Myopia group & Control group & $\boldsymbol{p}$ value \\
\hline Average thickness & $109.21 \pm 7.38(89-123)$ & $86.30 \pm 4.91(72-103)$ & 0.0001 \\
Superior thickness & $102.91 \pm 16.32(60-132)$ & $123.08 \pm 10.64(109-145)$ & 0.41 \\
Nasal thickness & $73.90 \pm 7.06(59-99)$ & $73.52 \pm 8.01(59-88)$ & 0.13 \\
Inferior thickness & $98.44 \pm 14.91(67-134)$ & $124.30 \pm 12.20(95-147)$ & 0.76 \\
Temporal thickness & $81.82 \pm 16.82(55-127)$ & $63.43 \pm 6.38(55-76)$ & 0.001 \\
\hline
\end{tabular}

$R N F L$ retinal nerve fiber layer 
eyes (more than 6.00 D of myopia), and moderate and low myopic eyes (less than $6.00 \mathrm{D}$ of myopia), which is consistent with the results observed in the current study, and in another study [15].

Previous studies showed that without magnification adjusted global and non-temporal RNFLs measurements, they appear significantly thinner than normal, in myopic eyes [18-20].

In the current study, a fair positive correlation was reported between spherical equivalent, and average $\mathrm{C} / \mathrm{D}$ area, vertical $\mathrm{C} / \mathrm{D}$ area, and inferior RNFL thickness $(p=0.006,0.0001$, and 0.001 ) respectively (Table 3 ). Spherical equivalent is liable to variability, as changes in corneal and lens refractive power, could affect refractometry, but not likely the axial length. Correlation between axial length and adjusted OCT measurements (RNFL, disc area and rim area) was not evaluated, as AL is used as a value in the magnification correction formula, so statistical artifacts may result, although some previous studies using OCT magnification corrected parameters studied such correlation. One study [13], reported a positive correlation between $\mathrm{AL}$, and the adjusted global RNFL thickness, but in another study [21], there was no significant correlation reported between $\mathrm{AL}$ and adjusted global RNFL or the adjusted ONH size.

$\mathrm{ONH}$ measurements derived from SD-OCT devices are reported to be more accurate and reproducible in evaluating highly myopic

Table 3 Significant correlations between spherical equivalent and $\mathrm{ONH}$ parameters and RNFL thickness in myopia group

\begin{tabular}{lll}
\hline & \multicolumn{2}{l}{ Spherical equivalent } \\
\cline { 2 - 3 } $\boldsymbol{R}$ & $\boldsymbol{p}$ value \\
\hline Average C/D & $0.292^{* *}$ & 0.006 \\
Vertical C/D & $0.444^{* *}$ & 0.0001 \\
Average RNFL & $0.214^{*}$ & 0.048 \\
Inferior RNFL & $0.341^{* *}$ & 0.001 \\
\hline
\end{tabular}

Grades for correlation $(r)$ : $0.00-0.24$ (weak or no association), 0.25-0.49 (fair association), 0.50-0.75 (moderate association), and $>0.75$ (strong association)

* Significant correlation; ${ }^{* *}$ Highly significant correlation patients, rather than depending on RNFL thickness measurements, as some anatomical optic disc changes, of highly myopic eyes, such as: tilting, oval configuration, and peripapillary atrophy [22], may influence the disc margin definition algorithms, potentially introducing some random bias to the RNFL thickness measurements.

Also, quantitative assessment of $\mathrm{ONH}$ parameters using SD-OCT, can be easily determined, due to high contrast between the non-reflective vitreous and the inner-limiting membrane, and the ability of SD-OCT to detect the end of Bruch's membrane [23], thereby defining an accurate reference plane for measuring the neuroretinal rim.

The magnification-adjusted measurements are used for accurate assessment of RNFL thickness and optic disc parameters in highly myopic eyes, and to minimize the misdiagnosis of glaucoma in such eyes, as patients with myopia have an increased risk of developing glaucoma.

Limitations to this study include: the relative small size of the study population, and the study included only Egyptian participants, so care should be taken on application of our results to different ethnicities. The SD OCT machine (Cirrus version 4.0), which was the only available machine during the study period, may have a few fallacies in detecting $\mathrm{BMO}$ and ILM around the ONH. More recent versions (Cirrus OCT Version 6.0) were therefore introduced to correct these inaccuracies.

\section{CONCLUSION}

The results of the current study indicate that there is a significant difference between highly myopic eyes and normal population, regarding ONH parameters and RNFL thickness as measured by SD-OCT. As OCT magnification-adjusted ONH parameters were larger, global and temporal RNEL were thicker in the myopia group, those magnification-adjusted parameters helped in accurate evaluation of ONH and RNFL in highly myopic eyes, in order to avoid misdiagnosis of glaucoma in such eyes. 


\section{ACKNOWLEDGEMENTS}

No funding or sponsorship was received for this study or publication of this article. All named authors meet the International Committee of Medical Journal Editors (ICMJE) criteria for authorship for this manuscript, take responsibility for the integrity of the work as a whole, and have given final approval to the version to be published.

Disclosures. Heba Radi AttaAllah, Ismail Ahmed Nagib Omar and Ahmed Shawkat Abdelhalim have nothing to disclose.

Compliance with Ethics Guidelines. The study has gained approval from the Local Research Ethics Committee, Faculty of Medicine, Minia University Hospitals. It is in accordance with the Helsinki Declaration of 1964, as revised in 2013. Informed consent was obtained from all patients for being included in this study.

Data Availability. The datasets during and/ or analyzed for the current study are available from the corresponding author on reasonable request.

Thanking Patient Participants. Authors would like to thank all patients who participated in this study.

Open Access. This article is distributed under the terms of the Creative Commons Attribution-NonCommercial 4.0 International License (http://creativecommons.org/licenses/ by-nc/4.0/), which permits any noncommercial use, distribution, and reproduction in any medium, provided you give appropriate credit to the original author(s) and the source, provide a link to the Creative Commons license, and indicate if changes were made.

\section{REFERENCES}

1. Jonas JB, Budde WM, Panda Jonas S. Ophthalmoscopic evaluation of the optic nerve head. Surv Ophthalmol. 1999;43:293-320.
2. Vessani RM, Moritz R, Batis L, et al. Comparison of quantitative imaging devices and subjective optic nerve head assessment by general ophthalmologists to differentiate normal from glaucomatous eyes. J Glaucoma. 2009;18:253-61.

3. DeLeon Ortega JE, Sakata LM, Kakati B, et al. Effect of glaucomatous damage on repeatability of confocal scanning laser ophthalmoscope, scanning laser polarimetry, and optical coherence tomography. Investig Ophthalmol Vis Sci. 2007;48:1156-63.

4. Samarawickrama C, Hong T, Jonas JB, Mitchell P. The measurement of normal optic nerve head parameters. Surv Ophthalmol. 2012;57(4):317-36.

5. Alencar LM, Zangwill LM, Weinreb RN, et al. Agreement for detecting glaucoma progression with the GDx guided progression analysis, automated perimetry, and optic disc photography. Ophthalmology. 2010;117:462-70.

6. Tay E, Seah SK, Chan SP, et al. Optic disk ovality as an index of tilt and its relationship to myopia and perimetry. Am J Ophthalmol. 2005;139:247-52.

7. Jonas JB. Optic disk size correlated with refractive error. Am J Ophthalmol. 2005;139:346-8.

8. Shoji T, Sato H, Ishida M, Takeuchi M, Chihara E. Assessment of glaucomatous changes in subjects with high myopia using spectral domain optical coherence tomography. Investig Ophthalmol Vis Sci. 2011;52(2):1098-102.

9. Littmann H. Determination of the real size of an object on the fundus of the living eye (in German). Klin Monatsbl Augenheilkd. 1982;180:286-9.

10. Leung CK, Cheng AC, Chong KK, et al. Optic disc measurements in myopia with optical coherence tomography and confocal scanning laser ophthalmoscopy. Investig Ophthalmol Vis Sci. 2007;48:3178-83.

11. Bennett AG, Rudnicka AR, Edgar DF. Improvements on Littmann's method of determining the size of retinal features by fundus photography. Graefes Arch Clin Exp Ophthalmol. 1994;232:361-7.

12. Feuer WJ, Budenz DL, Anderson DR, et al. Topographic differences in the age-related changes in the retinal nerve fiber layer of normal eyes measured by Stratus optical coherence tomography. J Glaucoma. 2011;20:133-8.

13. Kang SH, Hong SW, Im SK, Lee SH, Ahn MD. Effect of myopia on the thickness of the retinal nerve fiber layer measured by Cirrus HD optical coherence tomography. Investig Ophthalmol Vis Sci. 2010;51:4075-83. 
14. Xu L, Wang Y, Yang H, Zhang L, Jonas JB. Size of the neuroretinal rim and optic cup and their correlations with ocular and general parameters in adult Chinese: the Beijing eye study. Br J Ophthalmol. 2007;91:1616-9.

15. Hsu SY, Chang MS, Ko ML, Harnod T. Retinal nerve fiber layer thickness and optic nerve head size measured in high myopes by optical coherence tomography. Clin Exp Optom. 2013;96(4):373-8.

16. Hwang YH, Kim YY. Correlation between optic nerve head parameters and retinal nerve fibre layer thickness measured by spectral-domain optical coherence tomography in myopic eyes. Clin Exp Ophthalmol. 2012;40(7):713-20.

17. Ikuno $\mathrm{Y}$, Jo $\mathrm{Y}$, Hamasaki $\mathrm{T}$, Tano Y. Ocular risk factors for choroidal neovascularization in pathologic myopia. Investig Ophthalmol Vis Sci. 2010;51:3721-5.

18. Kim MJ, Lee EJ, Kim TW. Peripapillary retinal nerve fibre layer thickness profile in subjects with myopia measured using the Stratus optical coherence tomography. Br J Ophthalmol. 2010;94:115-20.

19. Vernon SA, Rotchford AP, Negi A, Ryatt S, Tattersal C. Peripapillary retinal nerve fibre layer thickness in highly myopic Caucasians as measured by Stratus optical coherence tomography. Br J Ophthalmol. 2008;92:1076-80.

20. Rauscher FM, Sekhon N, Feuer WJ, Budenz DL. Myopia affects retinal nerve fiber layer measurements as determined by optical coherence tomography. J Glaucoma. 2009;18:501-5.

21. Savini G, Barboni P, Parisi V, Carbonelli M. The influence of axial length on retinal nerve fibre layer thickness and optic-disc size measurements by spectral-domain OCT. $\mathrm{Br} \mathrm{J}$ Ophthalmol. 2012;96:57-61.

22. Tong L, Chan YH, Gazzard G, et al. Heidelberg retinal tomography of optic disc and nerve fiber layer in Singapore children: variations with disc tilt and refractive error. Investig Ophthalmol Vis Sci. 2007;48:4939-44.

23. Downs JC, Yang H, Girkin C, et al. Three-dimensional histomorphometry of the normal and glaucomatous monkey optic nerve head: neural canal and subarachnoid space architecture. Investig Ophthalmol Vis Sci. 2007;48:3195-208. 